\title{
Incorporation of Neurobiological Aspects of Aplysia's Associative Conditioning in Neural Networks for on-line Pattern Detection*
}

\author{
M.H. Spigt ${ }^{1}$, D.S. Brée ${ }^{1}$, M. Nielen ${ }^{2}$ \\ 1Univerisity of Manchester, Oxford Road, Manchester M13 9PL, UK, email: spigtt@cs.man.ac.uk \\ 2Utrecht University, PO box 80151, 3508 TD Utrecht, The Netherlands
}

In this study anatomical characteristics of a neurobiological model of associative conditioning (i.e. Aplysia, a marine mollusc) were used for the development of a novel neural network architecture for the recognition (and on-line classification) of temporal patterns. Compared to the performance of a standard Back Propagation network, the results indicate that this novel architecture is a step forward towards the construction of a reliable mechanism for time-series analysis.

The startingpoint was a standard 3-layer fully connected feed-forward architecture, consisting of 24 input units, 3 hidden units and 1 output unit. In experiment 2 and 3, direct connections between the last 12 input units and respectively only the output unit and only the hidden layer were defined. These connections were disabled for training. The input layer was divided into two independent parts, both of 12 units. This division opened the opportunity of introducing spatial and temporal independence in the input. While the first 12 units cover characteristics of $t=-1$, the second 12 units cover characteristics of $\mathrm{t}=0$. Following this procedure the input to the first 12 units can be compared with the 'to be Conditioned Stimulus' in the Aplysia model, and the second 12 units can be compared with the 'Unconditioned Stimulus' in the Aplysia model, while the output is defined along the 'Unconditioned Stimulus'. Then, by testing the network by stimulating only the first 12 units, an enhanced output would be expected.

In order to test the proposed neural network approach, a dairy herd environment was chosen. Mastitis, an udder infection, causes a qualitative and quantitative loss of milk. An important variable that can be measured during milking is the electrical conductivity (EC) of the milk. When a teat of the udder, a guarter, is infected, the composition of the milk changes, and therefore the EC in that quarter.

Quarter EC-series, measured every 5 seconds during the morning- and evening-milkings, were used for the construction of datasets. It was decided to use only the first 12 elements of an EC-series, because a lot of information is located in the beginning of EC-series.

The trainingset was constructed as follows: 12 measurements of EC-data of the morning-milking of the day before clinical mastitis was observed (i.e. $t=-1$ ) were combined with the 12 measurements of ECdata of morning-milking of the day clinical mastitis was actually observed (i.e. $t=0)$. The trainingset consisted of 51 healthy and 11 mastitic EC-series.

The testsets consisted of both healthy and (sub)-clinical EC-series: I: data of sub-clinical EC-series from the morning milking of the day before clinical mastitis was observed $(t=-1)$. II: data from the evening milking of that day $(t=-1)$. III: data of clinical EC-series from the morning milking of the day on which clinical mastitis was actually observed $(t=0)$. IV: data from the evening milking of that day. ( $t$ $=0$ )

Experiment 1. Standard Back Propagation architecture with no extra connections.

Experiment 2 . See experiment 1, except that for the second 12 units only to the output unit connections exist with a fixed value of 0.01 .

Experiment 3. See experiment 1, except that for the second 12 units only to the hidden units connections exist with a fixed value of 0.01 .

TABLE 1. The values indicate the number of correct or faulty classified EC-series.

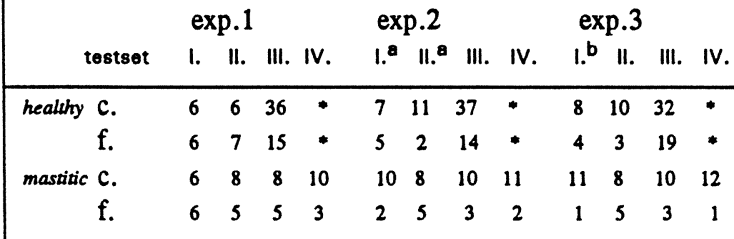

c.: correct classification, f.: faulty classification, *: not available.

\footnotetext{
- This study was sponsored by SKBS. SKBS (foundation for knowledge based systems) is a Dutch foundation that stimulates research in the field of KBS and technology transfer between universities and industry

a significant at $5 \%$ level for testset $I$ and II; I: $\mathcal{U}=2.84$, p-value 0.04 (Fisher's exact test); II: $U=4.06, p$-value 0.04

b significant at $1 \%$ level for testset I: $\mathcal{U}=6.40$, p-value 0.005 (Fisher's exact lest).
} 